\title{
Resting-State Network Functional Connectivity Patterns Associated with the Mindful Attention Awareness Scale
}

\author{
Elena Bilevicius, ${ }^{1,2}$ Stephen D. Smith, and Jennifer Kornelsen ${ }^{1-4}$
}

\begin{abstract}
Mindfulness refers to attending to moment-to-moment experiences with acceptance and no judgment. Several scales have been developed to quantify different components of mindfulness. The Mindful Attention Awareness Scale (MAAS) is particularly sensitive to trait mindfulness and is proposed to measure the attentional component of mindfulness. The purpose of this study was to identify the neural correlates of the MAAS in four resting-state networks related to attention - the default mode network (DMN), the salience network (SN), and the left and right central executive network (CEN). Thirty-two university students naive to mindfulness completed the MAAS and later underwent a resting-state functional magnetic resonance imaging scan. Resting-state data were analyzed using an independent component analysis; the scores from the MAAS were covaried to the connectivity maps in an analysis of covariance. The results indicate that variations in MAAS scores correlated with variations in functional connectivity patterns in resting-state networks. Specifically, within the SN and CEN, the MAAS was negatively correlated with functional connectivity in the precuneus, even though the precuneus is a key component of the DMN. Negative correlations in the DMN between the MAAS and the insula and negative correlations in the SN between the MAAS and the posterior cingulate cortex were also observed. These results suggest that MAAS scores (1) are correlated with the functional connectivity of several brain structures related to attention and (2) involve cross-network functional connectivity.
\end{abstract}

Keywords: fMRI; functional connectivity; Mindful Attention Awareness Scale; resting state; trait mindfulness

\section{Introduction}

$\mathbf{M}$ INDFULNESS IS DEFINED as purposeful attention to moment-to-moment experiences with an accepting and nonjudgmental stance (Brown and Ryan, 2003; KabatZinn, 1994, 2003). Mindfulness originates from Eastern traditions grounded in Buddhist meditative practice (Kabat-Zinn, 1994; Thera, 2014); however, in more recent years, mindfulness has been introduced into Western cultures in a more secular form. Various mindfulness-based programs exist to teach individuals the skills and techniques required to practice mindfulness. Indeed, it has been implemented as a noninvasive training course or treatment for various healthy (Goodman and Schorling, 2012) and patient populations, including anxious (Goldin et al., 2009) and depressed individuals (Hofmann et al., 2010), as well as experimentally induced (Bilevicius et al., 2016; Lutz et al., 2013; Zeidan et al., 2015) and chronic pain (Kabat-Zinn et al., 1985; la Cour and Petersen, 2015).
Mindfulness can be further divided into two broad categories: state and trait mindfulness. State mindfulness is a measure of how mindful someone is in the present moment and reflects a more transient, moment-to-moment experience of mindfulness (Tanay and Bernstein, 2013). Trait mindfulness, in contrast, is defined as an individual's natural or innate mindfulness tendency, which has been perceived as a stable, permanent characteristic (Brown and Ryan, 2003). The construct of trait mindfulness is multifaceted, including the ability to attend to and describe current experiences, the ability to have focused and sustained attention (Baer et al., 2004), and the ability to have continuous openness and receptive awareness to these experiences (Brown and Ryan, 2003).

One validated and commonly used measure of trait mindfulness is the Mindful Attention Awareness Scale (MAAS; Brown and Ryan, 2003). The MAAS is proposed to be a one-dimensional scale that measures the attentional component of mindfulness, but not the nonjudgmental component

\footnotetext{
${ }^{1}$ Department of Physiology and Pathophysiology, University of Manitoba, Winnipeg, Canada.

${ }^{2}$ St. Boniface Hospital Research, Winnipeg, Canada.

${ }^{3}$ Department of Psychology, University of Winnipeg, Winnipeg, Canada.

${ }^{4}$ Department of Radiology, University of Manitoba, Winnipeg, Canada.
} 
(Brown and Ryan, 2003). Given the frequency with which the MAAS is used in empirical studies (e.g., Cho et al., 2016; Shin et al., 2016), a thorough understanding of the neural structures-and networks of neural structures-associated with MAAS scores would be beneficial.

Functional neuroimaging has allowed researchers to delineate the neural substrates of many of the characteristics related to trait mindfulness, including the attentional components measured by the MAAS. Indeed, cortical and subcortical regions of the brain have been linked with attentional, emotional-regulation, and sensory-integration functions that make up the multifaceted trait of mindfulness (Creswell et al., 2007; Ives-Deliperi et al., 2011). For example, a task-based study by Creswell and colleagues (2007) revealed that the MAAS was associated with greater activity in the medial prefrontal cortex (mPFC). They concluded that the negative relationship observed between mPFC and amygdala activation was due to the increased attention involved in their affect-labeling task. This finding demonstrates that the MAAS is able to tap into the attentional component of mindfulness and suggests that focused attention is important in trait mindfulness.

The neural substrates of the attentional component of mindfulness are not limited to the $\mathrm{mPFC}$ and amygdala, however. Activity in the anterior cingulate cortex (ACC) has been linked to both attentional and emotional processes (Bush et al., 2000; Veen and Carter, 2006). Indeed, Ives-Deliperi and colleagues (2011) found that participants who underwent a mindfulness intervention and scored high on the Five-Facet Mindfulness Questionnaire (FFMQ; Baer et al., 2006) had a reduction in ACC activity. Given that three of the five factors assessed by the FFMQ involve attention in some form (observe, act with awareness, and nonreact items), this altered ACC response is noteworthy. Finally, increased posterior cingulate cortex (PCC) is associated with present moment awareness during meditation (Ives-Deliperi et al., 2011) Taken together, these results suggest that state mindfulness-corroborated by FFMQ scores-is associated with altered patterns of activity in several brain regions associated with attention, including the mPFC, amygdala, ACC, and PCC. However, less is known about the neural substrates underlying the attentional component of trait mindfulness.

Many of the neural structures that are active during taskbased studies of mindfulness are also key nodes in some of the brain's resting-state networks (Brewer et al., 2011; Gard et al., 2012; Ives-Deliperi et al., 2011). Therefore, an examination of resting-state networks could provide important insights into the neural substrates of trait mindfulness. Resting-state networks comprise brain structures with activity that is highly correlated over a period of time when the brain is at rest; this is referred to as functional connectivity. The best-known restingstate network is the default mode network (DMN), which comprises the mPFC, lateral parietal cortex, PCC, and precuneus (Christoff et al., 2016; Raichle, 2015; Raichle et al., 2001). The DMN is functionally active when an individual is not attending to an external stimulus or performing a demanding cognitive task. It is also active during mind wandering, which is essentially the opposite of being in a focused, mindful state (Beckmann et al., 2005; Fox and Raichle, 2007).

Previous studies have identified the neural correlates of trait mindfulness within the DMN. For example, Wang and colleagues (2014) used a region-of-interest (ROI) analysis to identify the role of the DMN in relation to trait mindfulness and found weakened functional connectivity between the thalamus and PCC. In a post hoc graph-based analysis, they found that all of the graph-based relationships for the significant negative relationship of the DMN and trait mindfulness were predictive for the thalamus, not the PCC. Another study that examined the relationship between the DMN and trait mindfulness in older adults found that trait mindfulness was associated with increased functional connectivity between the dorsal PCC and the precuneus (Shaurya Prakash et al., 2013). Finally, a study by Kong and colleagues (2016) reported that trait mindfulness was positively correlated with regional homogeneity of activity in the right insula, left parahippocampal gyrus, and left orbitofrontal cortex; it was negatively correlated with regional homogeneity in the right inferior frontal gyrus, an area related to attentional control and response inhibition. Together, these studies suggest that the functional connectivity of brain regions related to attention is likely altered as a function of trait mindfulness. The diverse regions of activity also suggest that an analysis of additional brain networks would provide novel insight into how mindfulness influences attentional functioning.

In this study, we examined the relationship between trait mindfulness, as measured by the MAAS, and four prominent resting-state networks: the DMN, the salience network (SN), and the left and right central executive networks (ICEN and rCEN). The ICEN and rCEN are characterized by functional connectivity between groups of neurons in the dorsal lateral PFC and are associated with cognitive tasks such as decisionmaking and information processing (Rosazza and Minati, 2011; Seeley et al., 2007), both of which are important for the attentional component of mindfulness. Although functionally and anatomically similar, the ICEN and rCEN often appear as separate components (networks) when resting-state data are analyzed. The $\mathrm{SN}$ has been defined by activity within the insula and ACC (Menon, 2015) and is important for the integration of sensory information (Seeley et al., 2007). It has recently been proposed to be a "switch" between the activation of the DMN and the CEN (Goulden et al., 2014; Menon, 2015). In mindfulness, detecting and attending to important, salient information is referred to as purposeful attention; this process is likely influenced by the function of the $\mathrm{SN}$. Given that the functions of the SN and CEN both have clear relevance to mindfulness, an examination of how the functional connectivity of these networks relates to trait mindfulness will provide novel information about the neural substrates of this personality characteristic.

Examining how functional connectivity is influenced by MAAS scores will also help corroborate the view that the MAAS is sensitive to the attentional components of mindfulness. Although the current research utilizes an independent component analysis (ICA) approach to resting-state data analysis-an approach that is data driven and "model free"-our examination of previous functional magnetic resonance imaging (fMRI) investigations of mindfulness does allow us to develop several hypotheses. Based on the literature, the interoceptive portion of mindfulness should be reflected as a negative correlation between MAAS scores and functional connectivity in the PFC within the DMN (Brewer et al., 2011; Josipovic, 2014). The awareness portion of mindfulness should be reflected as a positive correlation between MAAS scores and functional connectivity in the two main nodes of the SN, the ACC (Manna et al., 
2010) and the insula (Hasenkamp and Barsalou, 2012). This would be consistent with the awareness function of this network (Menon, 2015). Finally, the focused attention component of mindfulness should be represented as positive correlations between the MAAS and the functional connectivity of frontal regions of the brain that make up the lCEN and rCEN (Hasenkamp and Barsalou, 2012).

\section{Materials and Methods}

\section{Participants}

Thirty-two nonmeditating students enrolled in Introductory Psychology at The University of Winnipeg participated in the study (10 males, 22 females; mean age $=18.16$ years; $\mathrm{SD}=1.08$; age range 18-22 years). Data surrounding their awareness of mindfulness from popular culture are unknown; however, the aim of the study is to understand trait mindfulness, which is an intrinsic representation that should be unaltered by media. All participants completed magnetic resonance safety screening before scanning. The study was approved by the University of Winnipeg Research Ethics Board and the National Research Council Ethics Board, and written informed consent was obtained from all participants before participation. Participants received a $\$ 25$ honorarium for their participation.

\section{Psychological measures}

During a session before the scanning date, participants completed the MAAS (Brown and Ryan, 2003). By definition, the MAAS is proposed to measure one of the two key components of mindfulness: attention. This 15-item questionnaire includes statements such as "I rush through activities without being really attentive to them" and "I find myself doing things without paying attention." Participants independently rated items on a 6-point Likert scale from 1 (almost always) to 6 (almost never). Items on the MAAS are reverse scored; higher scores indicate high trait mindfulness. The MAAS has been demonstrated to have good internal consistency $(\alpha=0.85)$ and test retest reliability ( $r=0.81)$ (Brown and Ryan, 2003).

\section{Study paradigm}

A 3D high-resolution anatomical magnetic resonance imaging was acquired for all participants after the initial localizer. After the anatomical scan, a 7-min resting-state scan was run to obtain the functional data. Participants were instructed to lay still with their eyes closed without falling asleep for the entire scanning session. All participants reported to have remained awake during the scanning session.

\section{Data acquisition}

Data were collected using a 3T Siemens TRIO MRI scanner (Siemens, Erlangen, Germany). For coregistration of functional data, high-resolution T1-weighted gradient-echo images were acquired using an MP-RAGE sequence $(1 \mathrm{~mm}$ slice thickness, 0 gap, repetition time $[\mathrm{TR}] /$ echo time $[\mathrm{TE}]=1900 /$ $2.2 \mathrm{msec}$, in plane resolution $0.94 \times 0.94 \mathrm{~mm}, 256 \times 256$ matrix, field of view [FOV] $24 \mathrm{~cm}$ ).

The fMRI component of the study was collected using conventional blood oxygenation level dependent imaging techniques. Resting-state fMRI data were acquired with a whole brain echo planar imaging sequence. This 7-min scan con- sisted of 140 volumes and was acquired using the following parameters: $3 \mathrm{~mm}$ slice thickness, 0 gap, TR/TE $=3000 /$ $30 \mathrm{msec}$, flip angle $=90^{\circ}, 64 \times 64$ matrix, FOV $24 \mathrm{~cm}$.

\section{Data analysis}

Imaging data preprocessing and statistical analyses were performed with BrainVoyager QX 2.8 software (Brain Innovation BV, Maastricht, The Netherlands). The preprocessing of functional data began with a trilinear/sync interpolation 3D motion correction. Motion in the three translations and three rotations was then regressed out from the data before further analyses. Preprocessing included slice scan correction, temporal filtering, and spatial smoothing $(8 \mathrm{~mm}$ full-width halfmaximum Gaussian filter), followed by coregistration of the functional data to the anatomical data in native space. The high-resolution anatomical data were manually spatially normalized by warping the data into the standardized Talairach space. After the normalization, the anatomical and preprocessed functional data were linked and the functional volume time course data were warped to Talairach space.

Single-subject ICA was carried out with the fast ICA algorithm (Hyvarinen and Oja, 2000) for all 32 participants to reveal correlated neural activity fluctuations. During this initial step, 20 independent components (ICs) for all 32 subjects were extracted from the data. To perform a grouped analysis, a group-level ICA was completed using the self-organizing group-level ICA (Sog-ICA; Esposito et al., 2005) plugin. In this step, the most similar ICs for all 32 participants were clustered in a hierarchical process at the group level, resulting in a final set of 20 ICs. These 20 components were visually inspected to identify the DMN, SN, ICEN, and rCEN. These networks were verified by ensuring correspondence between the Talairach coordinates of the functionally connected voxels in our data with brain regions identified in previous studies for each network (Rosazza and Minati, 2011; Shirer et al., 2012). The four IC maps were converted to volumes of interest (VOIs), which provided output including the peak coordinate of each cluster within each IC, the number of active voxels in each cluster, and the $t$-value of the observed clusters. This output was then entered into TalairachDaemon software (www.talairach.org/daemon.html), which provided the anatomical label for the peak coordinate of each cluster and the Brodmann areas (BAs), if applicable.

To determine the relationship between trait mindfulness and the functional connectivity in the four resting-state networks, individual scores from the MAAS were entered as covariates in an analysis of covariance (ANCOVA) for each network, that is, each of the four ICs. The four resulting brain maps show a correlation between MAAS scores and the average time series in every voxel in each IC map. These maps characterize the trait mindfulness representation in the DMN, SN, rCEN, and ICEN. The resulting cluster maps were corrected with a Monte Carlo cluster threshold estimator correction plugin at 1000 iterations, a technique used to reduce the likelihood of obtaining a false positive, and evaluated at $p=0.05$. Finally, these cluster maps were converted to VOIs, which provided output including the peak coordinate for each cluster in the maps, the number of active voxels in each cluster, and the $r$ value of the observed clusters. This output was then entered into Talairach-Daemon software (www.talairach.org/daemon .html), which provided the anatomical name of the peak coordinate of each cluster and the BAs, if applicable. 


\section{Results}

\section{Psychological measures}

The MAAS was scored according to its standardized method. Each individual score was recorded, with higher scores representing higher trait mindfulness. These scores were later used in the ANCOVA (mean =3.95; $\mathrm{SD}=0.56$; range $=2.73-5.07)$.

\section{Identification of resting-state networks}

The resting-state networks were identified in the grouplevel data before being covariated with mindfulness scores (Table 1). As already noted, the DMN and SN were identified as single components, whereas the CEN was detected as two separate components, the $1 \mathrm{CEN}$ and the rCEN (Fig. 1).

\section{DMN functional connectivity}

Data were analyzed in the four a priori networks already mentioned. Table 2 outlines the functionally connected brain regions that are correlated with the MAAS and their corresponding BAs, Talairach coordinates of the peak clus- ters, cluster sizes, and corresponding $r$ and $p$ values. Within the DMN, MAAS scores were positively correlated with functional connectivity in the right parahippocampal gyrus (BA 36), as well as in the left caudate and right medial frontal gyrus (MFG; BA 10; see Fig. 2). In contrast, MAAS scores were negatively correlated with functional connectivity in the left MFG (BA 32), left superior temporal gyrus (STG; BA 38), and left insula (BA 13). Of note, the MFG and STG are key components of the DMN, whereas the insula is a major component of the $\mathrm{SN}$.

\section{SN functional connectivity}

In the SN, left insula (BA 13) was positively correlated with the MAAS. The right cuneus cluster (BA 18) was the largest and most significant cluster that was negatively correlated with the MAAS. This cluster encompasses the precuneus, which is a key component of the DMN (Fig. 2).

\section{Central executive network functional connectivity}

The Sog-ICA identified two separate lateralized CEN components, the 1CEN and the rCEN. These components

Table 1. Talairach Coordinates of Resting-State Networks

\begin{tabular}{|c|c|c|c|c|c|c|c|c|}
\hline \multirow[b]{2}{*}{ Region } & \multirow[b]{2}{*}{ Hemisphere } & \multirow[b]{2}{*}{ Gyrus } & \multicolumn{4}{|c|}{ Talairach coordinates } & \multirow[b]{2}{*}{ Cluster size } & \multirow[b]{2}{*}{$t$} \\
\hline & & & $B A$ & $X$ & $Y$ & $Z$ & & \\
\hline \multicolumn{9}{|l|}{ DMN } \\
\hline \multirow[t]{2}{*}{ Limbic } & Left & Cingulate & 31 & -3 & -40 & 31 & 45602 & 14.30 \\
\hline & Left & Parahippocampal & & -21 & -16 & -14 & 2121 & 6.81 \\
\hline \multirow{2}{*}{ Frontal } & Right & Inferior frontal & & 48 & 5 & 22 & 794144 & 4.60 \\
\hline & Left & Medial frontal & 10 & -3 & 53 & 10 & 118250 & 12.71 \\
\hline \multirow[t]{3}{*}{ Temporal } & Right & Middle temporal & & 57 & 8 & -23 & 22622 & 7.34 \\
\hline & Left & Middle temporal & & -60 & -10 & -14 & 30727 & 7.87 \\
\hline & Left & Middle temporal & & -48 & -64 & 28 & 19655 & 8.79 \\
\hline Parietal & Right & Angular & & 45 & -61 & 31 & 6232 & 7.02 \\
\hline Sublobar & Left & Thalamus & & -3 & -10 & 7 & 763 & 4.31 \\
\hline \multicolumn{9}{|l|}{$\mathrm{SN}$} \\
\hline \multirow[t]{2}{*}{ Limbic } & Right & Uncus & 38 & 21 & 8 & -38 & 802 & 3.99 \\
\hline & Left & Parahippocampal & & -21 & -40 & 4 & 1305 & 4.86 \\
\hline \multirow[t]{3}{*}{ Temporal } & Right & Subgyral & & 24 & -43 & 1 & 1836 & 7.36 \\
\hline & Left & Inferior temporal & 20 & -60 & -16 & -20 & 660 & 4.26 \\
\hline & Right & Superior temporal & 22 & 69 & -37 & 14 & 789 & 4.05 \\
\hline Occipital & Right & Cuneus & & 21 & -67 & 10 & 1046 & 4.34 \\
\hline \multirow[t]{2}{*}{ Sublobar } & Left & Insula & & -30 & -10 & 16 & 269947 & 10.07 \\
\hline & Left & Subgyral & & -18 & -49 & -29 & 20888 & 6.13 \\
\hline \multicolumn{9}{|l|}{ rCEN } \\
\hline Frontal & Right & Middle frontal & & 42 & 23 & 47 & 82511 & 12.08 \\
\hline \multirow[t]{2}{*}{ Parietal } & Right & Supramarginal & & 60 & -49 & 31 & 51311 & 7.95 \\
\hline & Left & Inferior parietal & 40 & -45 & -46 & 40 & 1085 & 3.97 \\
\hline Posterior & Left & Tuber & & -33 & -67 & -29 & 8482 & 5.24 \\
\hline No gray matter found & & & & 48 & 8 & -36 & 551 & 4.71 \\
\hline \multicolumn{9}{|l|}{ 1CEN } \\
\hline Frontal & Left & Middle frontal & 8 & -42 & 23 & 47 & 60601 & 8.00 \\
\hline Temporal & Left & Inferior temporal & & -57 & -7 & -29 & 1670 & 4.45 \\
\hline Sublobar & Left & Extranuclear & & -15 & 5 & 19 & 315 & 3.80 \\
\hline Posterior & Right & Inferior semilunar lobule & & 30 & -70 & -41 & 2138 & 5.07 \\
\hline No gray matter found & & & & -67 & -37 & -11 & 52042 & 7.87 \\
\hline
\end{tabular}

Peak coordinates for the four resting-state networks. Cluster size notes number of active voxels within the cluster; $t$ statistic was significant at $p<0.001$.

BA, Brodmann area; DMN, default mode network; 1CEN, left central executive network; rCEN, right central executive network; SN, salience network. 
FIG. 1. Identification of resting-state networks (A) DMN, (B) SN, (C) rCEN, (D) ICEN on a Talairach brain $(N=32)$. DMN, default mode network; lCEN, left central executive network; rCEN, right central executive network; SN, salience network.
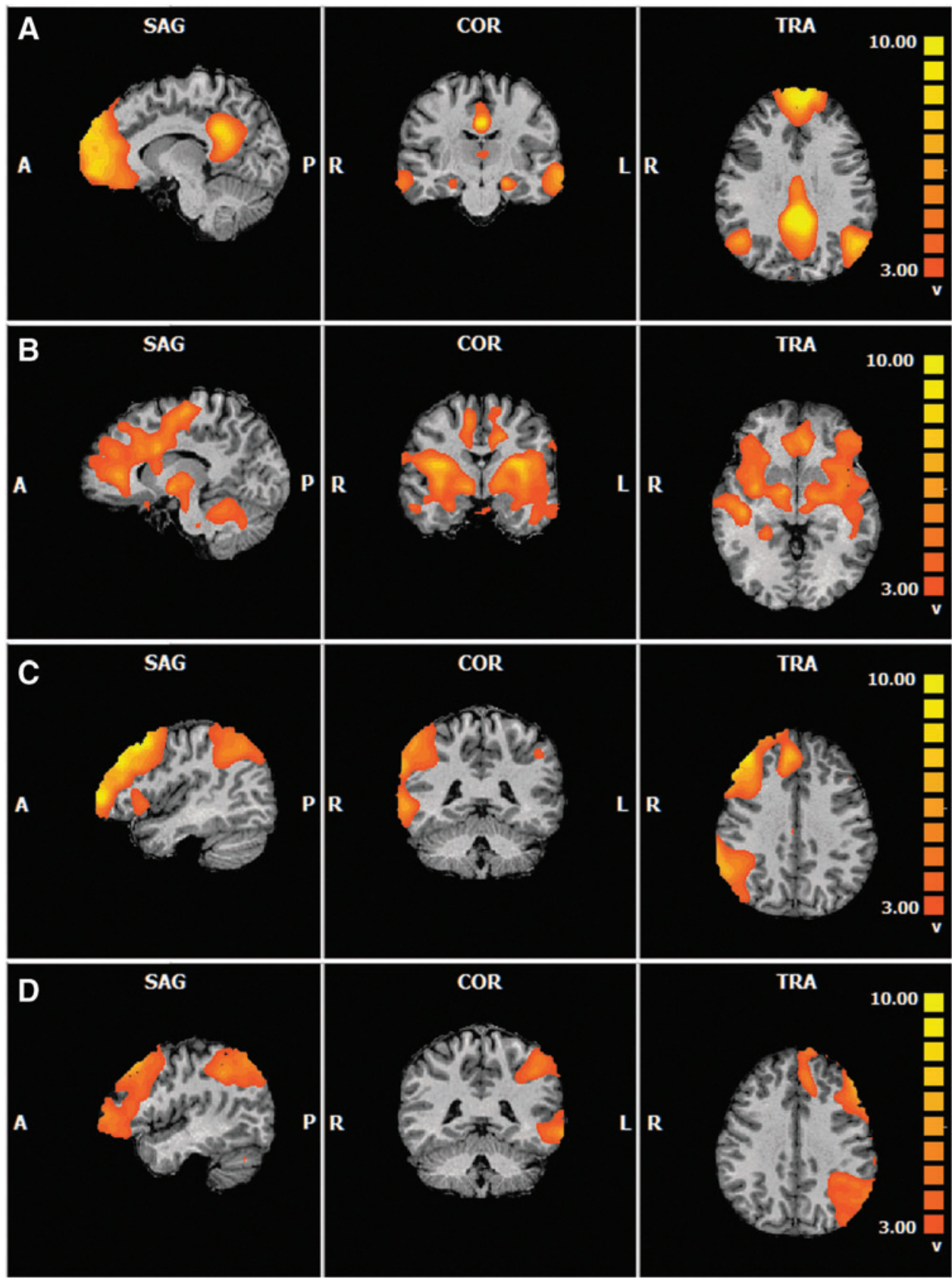

were run through separate ANCOVAs. The ANCOVA produced similar patterns of data for the ICEN and rCEN. The MAAS was positively correlated with functional connectivity in the right middle frontal gyrus in both the rCEN (BA 10/ 6) and 1CEN (BA 6; see Fig. 2). Negative correlations between the MAAS and functional connectivity in the left cuneus (BA 18) and precuneus (BA 31) were found in the rCEN and ICEN, respectively. It is interesting to note that key components of the DMN network appear to be correlated within both the rCEN and ICEN.

\section{Discussion}

This study examined how scores on the MAAS, a questionnaire sensitive to the attentional component of mindfulness, related to differences in the functional connectivity of four common resting-state networks. In the DMN, the MAAS was negatively correlated with functional connectivity in the left middle and left inferior frontal gyri. The $\mathrm{SN}$ revealed significant positive correlations of functional connectivity in the ACC and the left insula. Both the rCEN and 1CEN demonstrated positive correlations with functional connectivity in the right middle frontal gyrus. These data suggest that many of the brain regions whose functional connectivity covaries with MAAS scores are also involved in attention to moment-to-moment experiences.

The DMN is a resting-state network that has been associated with activity in brain regions, such as the medial frontal and STQ, lateral parietal cortex, PCC, and the precuneus (Buckner et al., 2008; Raichle, 2015). In this study, MAAS 
Table 2. Talairach Coordinates of Correlations Between Trait Mindfulness and Functional Connectivity of Resting-State Networks

\begin{tabular}{|c|c|c|c|c|c|c|c|c|c|}
\hline \multirow[b]{2}{*}{ Region } & \multirow[b]{2}{*}{ Hemisphere } & \multirow[b]{2}{*}{ Gyrus } & \multicolumn{7}{|c|}{ Talairach Coordinates } \\
\hline & & & $B A$ & $X$ & $Y$ & $Z$ & Cluster size & $\mathrm{r}$ & $\mathrm{p}$ \\
\hline \multicolumn{10}{|l|}{ DMN } \\
\hline Limbic & Right & Parahippocampal & 36 & 39 & -25 & -11 & 7012 & 0.62 & 0.000132 \\
\hline \multirow[t]{3}{*}{ Frontal } & Right & Medial frontal & 10 & 21 & 50 & 7 & 8106 & 0.54 & 0.001294 \\
\hline & Left & Medial frontal & 32 & -6 & 8 & 43 & 9654 & -0.66 & 0.000043 \\
\hline & Left & Subgyral & & -24 & 44 & 1 & 3032 & 0.49 & 0.004717 \\
\hline \multirow[t]{2}{*}{ Temporal } & Left & Superior temporal & 38 & -30 & 17 & -29 & 1806 & -0.47 & 0.006783 \\
\hline & Left & Caudate & & -39 & -40 & 4 & 3438 & 0.60 & 0.000264 \\
\hline Parietal & Left & Postcentral & 3 & -24 & -28 & 52 & 1793 & -0.50 & 0.003472 \\
\hline Sublobar & Left & Insula & 13 & -48 & 5 & 7 & 3531 & -0.62 & 0.000168 \\
\hline \multicolumn{10}{|l|}{$\mathrm{SN}$} \\
\hline Occipital & Right & Cuneus & 18 & 9 & -73 & 16 & 15708 & -0.70 & 0.000007 \\
\hline Temporal & Right & Inferior temporal & 20 & 63 & -19 & -23 & 4638 & -0.59 & 0.000357 \\
\hline Sublobar & Left & Insula & 13 & -42 & -40 & 25 & 4842 & 0.60 & 0.000279 \\
\hline No gray matter found & & & & 33 & 26 & 19 & 5650 & 0.55 & 0.001211 \\
\hline No gray matter found & & & & -30 & 14 & 19 & 5768 & 0.62 & 0.000176 \\
\hline \multicolumn{10}{|l|}{ rCEN } \\
\hline Limbic & Right & Cingulate & 23 & 6 & -25 & 28 & 2790 & 0.46 & 0.008698 \\
\hline \multirow[t]{3}{*}{ Frontal } & Right & Precentral & 9 & 45 & 23 & 37 & 4405 & 0.63 & 0.000105 \\
\hline & Right & Middle frontal & 10 & 43 & 47 & -2 & 6199 & 0.64 & 0.000081 \\
\hline & Left & Superior frontal & 6 & -12 & 20 & 52 & 2890 & -0.58 & 0.000449 \\
\hline Occipital & Left & Cuneus & 18 & -3 & -73 & 19 & 4867 & -0.55 & 0.001157 \\
\hline \multirow[t]{4}{*}{ Temporal } & Right & Supramarginal & 40 & 48 & -49 & 19 & 9232 & 0.59 & 0.000400 \\
\hline & Right & Middle temporal & 21 & 54 & -19 & -8 & 3444 & 0.68 & 0.000017 \\
\hline & Right & Caudate & & 36 & -31 & 4 & 2362 & -0.48 & 0.005011 \\
\hline & Left & Subgyral & & -39 & -34 & -2 & 13706 & -0.71 & 0.000006 \\
\hline Sublobar & Right & Caudate & & 18 & 20 & 13 & 16023 & -0.63 & 0.000112 \\
\hline \multirow[t]{2}{*}{ Posterior } & Left & Declive & & -24 & -67 & -20 & 6811 & 0.50 & 0.003339 \\
\hline & Left & Inferior semilunar & & 0 & -58 & -42 & 2786 & 0.57 & 0.000657 \\
\hline No gray matter found & & & & -27 & -10 & 37 & 9169 & -0.69 & 0.000013 \\
\hline \multicolumn{10}{|l|}{ 1CEN } \\
\hline \multirow[t]{3}{*}{ Frontal } & Right & Middle frontal & 6 & 30 & -7 & 55 & 17179 & 0.66 & 0.000039 \\
\hline & Left & Medial frontal & 10 & -12 & 59 & 7 & 9580 & -0.60 & 0.000252 \\
\hline & Left & Inferior temporal & & -51 & -10 & -23 & 2896 & -0.45 & 0.009705 \\
\hline \multirow[t]{2}{*}{ Parietal } & Left & Precuneus & 31 & -12 & -58 & 34 & 8784 & -0.61 & 0.000191 \\
\hline & Right & Precuneus & 7 & 27 & -64 & 28 & 17798 & 0.62 & 0.000166 \\
\hline \multirow[t]{2}{*}{ Sublobar } & Left & Caudate & & -18 & 11 & 13 & 4171 & 0.60 & 0.000313 \\
\hline & Right & Lentiform nucleus & & 21 & 5 & 10 & 5005 & 0.59 & 0.000403 \\
\hline No gray matter found & & & & -45 & -46 & 10 & 7946 & -0.67 & 0.000031 \\
\hline
\end{tabular}

Peak coordinates of resting-state networks covaried with MAAS scores.

MAAS, Mindful Attention Awareness Scale.

scores were negatively correlated with functional connectivity in the MFG, a key node of the DMN, which is consistent with existing literature (Doll et al., 2015; Hasenkamp and Barsalou, 2012). Interestingly, the medial frontal region of the DMN has also been linked with mind wandering (Buckner et al., 2008; Mason et al., 2007; Smallwood and Schooler, 2006, 2015). Mind wandering is often defined as the fluid shift from thoughts to events in the environment; mindfulness, in contrast, has been associated with present-moment awareness and focused attention (Brown and Ryan, 2003; Kabat-Zinn, 2003). The fact that MAAS scores were negatively correlated with functional connectivity in the DMN-particularly in the left MFG and left STG-is, therefore, consistent with these complementary areas of research.

The DMN can be divided into separate subcomponents: anterior DMN and posterior DMN. The main node of the anterior DMN is the mPFC, and its function is associated with self-referential processing (Andrews-Hanna et al., 2010). In contrast, the posterior DMN is made up of the PCC and precuneus, which are more associated with conscious processing and affective responses to environmental stimuli (Vogt and Laureys, 2005). In our DMN results, MAAS scores were negatively correlated with functional connectivity in the medial and middle frontal gyri. Looking across the results from all four networks, MAAS scores were negatively correlated with functional connectivity in the precuneus and cuneus within the $1 \mathrm{CEN}, \mathrm{rCEN}$, and $\mathrm{SN}$; in other words, key components of the DMN were negatively correlated with the functional connectivity of the other three networks. This is consistent with Doll and colleagues (2015), who found a significant decoupling between the anterior and posterior components of the DMN with relation to the MAAS. Going one step further, our results are also consistent with Hasenkamp and Barsalou (2012), who noted the 

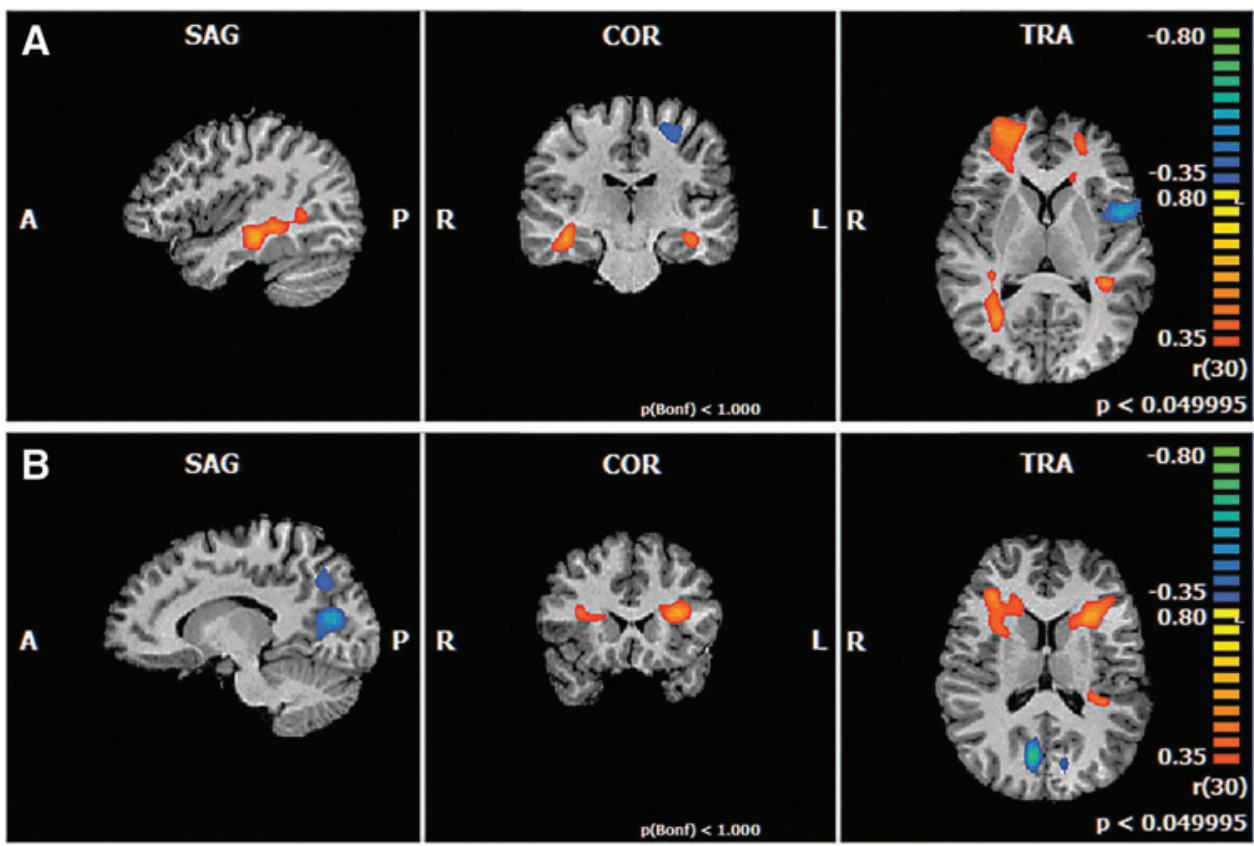

FIG. 2. Functional connectivity covaried with mindfulness scores in the (A) DMN, (B) $\mathrm{SN}$, (C) rCEN, (D) 1CEN on a Talairach brain. Displayed are the brain regions with positively correlated functional connectivity depicted with orange voxels and regions with negatively correlated functional connectivity depicted with blue voxels.
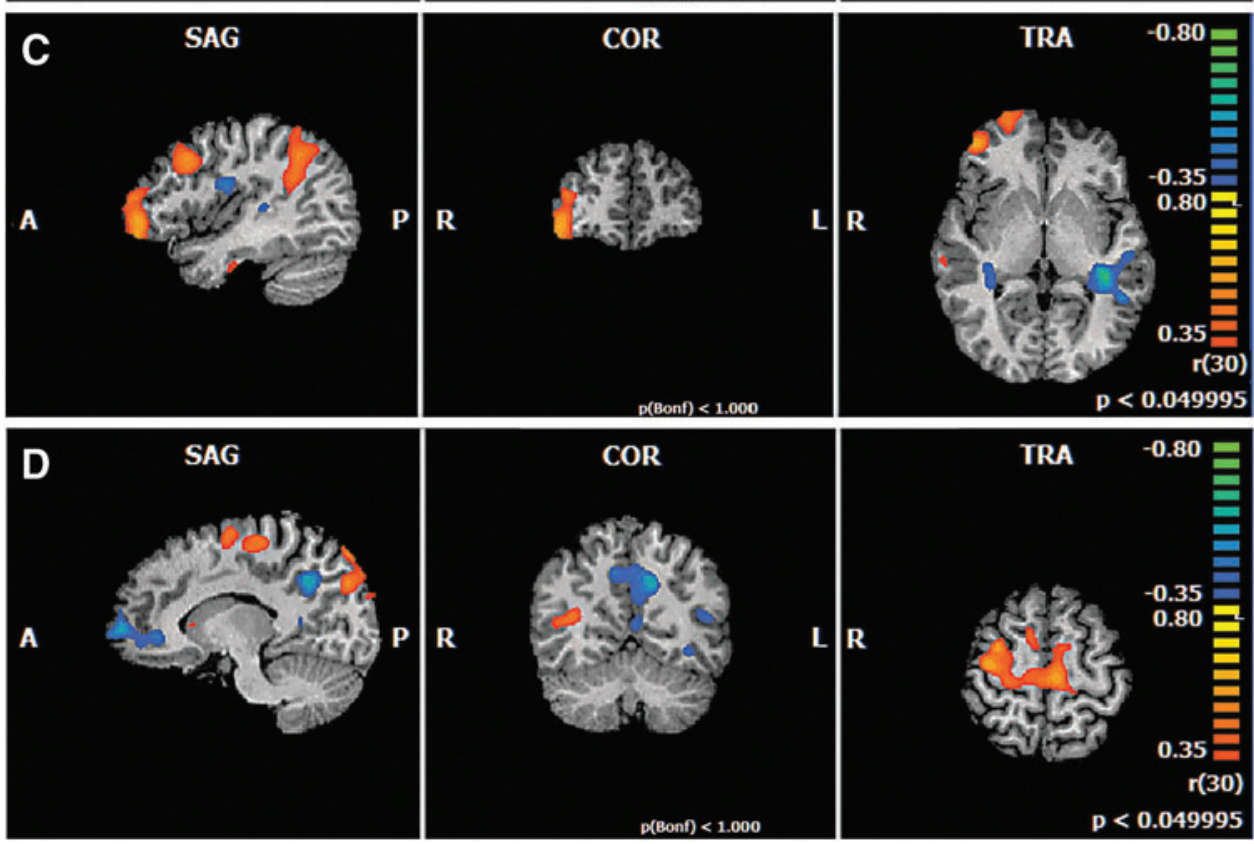

importance between the interaction of the DMN and CEN in the focusing on present experiences.

The SN has also been associated with mindfulness, specifically in terms of the integration of sensory information (Seeley et al., 2007). The SN has recently been proposed to play a role in the switching of activation between the DMN and CEN (Goulden et al., 2014; Sridharan et al., 2008). In terms of mindfulness, the SN may be important for the switch between less mindful states (e.g., mind wandering) and increased attention to sensations and experiences. The results of this study reveal that the MAAS is positively correlated with functional connectivity in the left ACC and negatively correlated with functional connectivity in the right precuneus. The increase in functional connectivity of the ACC is consistent with previous research, showing that meditation is related to increased theta activity in ACC neurons (Tang et al., 2009), increased density of white matter pathways related to the ACC (Tang et al., 2012), and an overall improvement in attentional control (Tang et al., 2007). The results of this research, therefore, indicate that individuals who have high trait mindfulness (as measured by the MAAS) have greater functional connectivity in a neural region that is altered by meditation training.

In this study, the CEN was divided into two separate components, the rCEN and the ICEN. Interestingly, the patterns of functional connectivity within the rCEN and ICEN were quite similar. Specifically, the MAAS was positively correlated with functional connectivity in the middle frontal gyrus in both the rCEN and ICEN, and negatively correlated with functional connectivity in the cuneus in the rCEN and precuneus in the 1CEN. The bilateral correlations observed here provide a novel finding about the potential relationship between mindfulness and executive functioning/attentional 
abilities. Future studies linking resting-state functional connectivity with behavioral assessments of executive functioning and attentional processing would help clarify this point.

It is worth noting the relationship of functional connectivity between various resting-state networks, or what is referred to as cross-network connectivity. Cross-network connectivity is commonly observed when key nodes of one network appear within a separate network (e.g., if the insula emerged in a functional connectivity map of the DMN). In the current data, crossnetwork connectivity was observed between the DMN and $\mathrm{SN}$ as well as between the DMN and CEN. However, this study is limited by the lack of quantitative measures of the cross-network functional connectivity. The use of the SogICA for producing the functional connectivity maps allowed us to detect all of the significant correlations in the brain's functional connectivity, rather than focusing solely on the connectivity of preselected seed regions. The original hypotheses surrounded the functional connectivity correlations within each network; however, we observed unanticipated crossnetwork functional connectivity results. As a result, this article can only report quantitative measures for the correlations of the MAAS and functional connectivity within each network and then speculate about the observed patterns of betweennetwork, cross-network, functional connectivity correlations.

There is controversy about the use of the MAAS as a measure of trait mindfulness (Medvedev et al., 2016; Van Dam et al., 2010). One of the main criticisms to arise is that the MAAS does not provide a complete definition of mindfulness (i.e., the nonjudgment component) and lacks the ability to accurately and adequately differentiate between varying levels of trait mindfulness. However, the developers of the MAAS noted that the focus of this scale is solely on the attention and awareness component of mindfulness (Brown and Ryan, 2003). As such, the conclusions about mindfulness drawn from this study focus on this component.

\section{Conclusion}

In conclusion, our study demonstrates that scores on the MAAS are correlated with specific patterns of functional connectivity in the DMN, SN, ICEN, and rCEN. The MAAS had negative correlations with functional connectivity of key components of the DMN and a positive correlation with functional connectivity of the ACC in the SN. Surprisingly, trait mindfulness was associated with a large amount of cross-network functional connectivity, which was not anticipated at the outset of this study. The results of this whole-brain data-driven approach will inform ROI hypothesis development for future seed-based analyses. Future research will compare the neural correlates obtained with the MAAS, which focuses on the attentional component, with those of another well-established mindfulness questionnaire, the FFMQ, which focuses on acting with awareness, and includes additional components such as nonjudging and nonreactivity.

\section{Acknowledgments}

The authors wish to thank the National Research Council Institute for Biodiagnostics radiology technical staff and Rachel Ganaden, Charley McDonald-Mitchell, and Theresa McIver for their assistance with data collection. This work was supported by the Natural Sciences and Engineering Research Council (NSERC) of Canada (grant no. 2014-002).

\section{Author Disclosure Statement}

This study has been approved by our institutional ethics committees and has been performed in accordance with the outlined ethical standards. All participants provided written, informed consent before their participation in the study. The authors declare that no competing financial interests exist. All procedures performed in studies involving human participants were in accordance with the ethical standards of the institutional and/or national research committee and with the 1964 Helsinki declaration and its later amendments or comparable ethical standards.

\section{References}

Andrews-Hanna JR, Reidler JS, Sepulcre J, Poulin R, Buckner RL. 2010. Functional-anatomic fractionation of the brain's default network. Neuron 65:550-562.

Baer RA, Smith GT, Allen KB. 2004. Assessment of mindfulness by self-report: the Kentucky inventory of mindfulness skills. Assessment 11:191-206.

Baer RA, Smith GT, Hopkins J, Krietemeyer J, Toney L. 2006. Using self-report assessment methods to explore facets of mindfulness. Assessment 13:27-45.

Beckmann CF, DeLuca M, Devlin JT, Smith SM. 2005. Investigations into resting-state connectivity using independent component analysis. Philos Trans R Soc Lond B Biol Sci 360:1001-1013.

Bilevicius E, Kolesar TA, Kornelsen J. 2016. Altered neural activity associated with mindfulness during nociception: a systematic review of functional MRI. Brain Sci 6. doi:10.3390/ brainsci6020014

Brewer JA, Worhunsky PD, Gray JR, Tang YY, Weber J, Kober H. 2011. Meditation experience is associated with differences in default mode network activity and connectivity. Proc Natl Acad Sci U S A 108:20254-20259.

Brown KW, Ryan RM. 2003. The benefits of being present: mindfulness and its role in psychological well-being. J Pers Soc Psychol 84:822-848.

Buckner RL, Andrews-Hanna JR, Schacter DL. 2008. The brain's default network: anatomy, function, and relevance to disease. Ann N Y Acad Sci 1124:1-38.

Bush G, Luu P, Posner MI. 2000. Cognitive and emotional influences in anterior cingulate cortex. Trends Cogn Sci 4:215-222.

Cho S, Lee H, Oh KJ, Soto JA. 2017. Mindful attention predicts greater recovery from negative emotions, but not reduced reactivity. Cogn Emot 31:1252-1259.

Christoff K, Irving ZC, Fox KC, Spreng RN, Andrews-Hanna JR. 2016. Mind-wandering as spontaneous thought: a dynamic framework. Nat Rev Neurosci 17:718-731.

Creswell JD, Way BM, Eisenberger NI, Lieberman MD. 2007. Neural correlates of dispositional mindfulness during affect labeling. Psychosom Med 69:560-565.

Doll A, Hölzel BK, Boucard CC, Wohlschläger AM, Sorg C. 2015. Mindfulness is associated with intrinsic functional connectivity between default mode and salience networks. Front Hum Neurosci 9:461.

Esposito F, Scarabino T, Hyvarinen A, Himberg J, Formisano E, Comani S, et al. 2005. Independent component analysis of fMRI group studies by self-organizing clustering. Neuroimage 25:193-205.

Fox MD, Raichle ME. 2007. Spontaneous fluctuations in brain activity observed with functional magnetic resonance imaging. Nat Rev Neurosci 8:700-711.

Gard T, Holzel BK, Sack AT, Hempel H, Lazar SW, Vaitl D, Ott U. 2012. Pain attenuation through mindfulness is associated 
with decreased cognitive control and increased sensory processing in the brain. Cereb Cortex 22:2692-2702.

Goldin P, Ramel W, Gross J. 2009. Mindfulness meditation training and self-referential processing in social anxiety disorder: behavioral and neural effects. J Cogn Psychother 23:242-257.

Goodman MJ, Schorling JB. 2012. A mindfulness course decreases burnout and improves well-being among healthcare providers. Int J Psychiatry Med 43:119-128.

Goulden N, Khusnulina A, Davis NJ, Bracewell RM, Bokde AL, McNulty JP, Mullins PG. 2014. The salience network is responsible for switching between the default mode network and the central executive network: replication from DCM. Neuroimage 99:180-190.

Hasenkamp W, Barsalou LW. 2012. Effects of meditation experience on functional connectivity of distributed brain networks. Front Hum Neurosci 6:38.

Hofmann SG, Sawyer AT, Witt AA, Oh D. 2010. The effect of mindfulness-based therapy on anxiety and depression: a meta-analytic review. J Consult Clin Psychol 78:169-183.

Hyvarinen A, Oja E. 2000. Independent component analysis: algorithms and applications. Neural Netw 13:411-430.

Ives-Deliperi VL, Solms M, Meintjes EM. 2011. The neural substrates of mindfulness: an fMRI investigation. Soc Neurosci 6:231-242.

Josipovic Z. 2014. Neural correlates of nondual awareness in meditation. Ann N Y Acad Sci 1307:9-18.

Kabat-Zinn J. 1994. Wherever You Go, There You Are: Mindfulness Meditation for Everyday Life. New York: Hyperion.

Kabat-Zinn J. 2003. Mindfulness-based interventions in context: past, present, and future. Clin Psychol 10:144-156.

Kabat-Zinn J, Lipworth L, Burney R. 1985. The clinical use of mindfulness meditation for the self-regulation of chronic pain. J Behav Med 8:163-190.

Kong F, Wang X, Song Y, Liu J. 2016. Brain regions involved in dispositional mindfulness during resting state and their relation with well-being. Soc Neurosci 11:331-343.

la Cour P, Petersen M. 2015. Effects of mindfulness meditation on chronic pain: a randomized controlled trial. Pain Med 16: 641-652.

Lutz A, McFarlin DR, Perlman DM, Salomons TV, Davidson RJ. 2013. Altered anterior insula activation during anticipation and experience of painful stimuli in expert meditators. Neuroimage 64:538-546.

Manna A, Raffone A, Perrucci MG, Nardo D, Ferretti A, Tartaro A, et al. 2010. Neural correlates of focused attention and cognitive monitoring in meditation. Brain Res Bull 82:46-56.

Mason MF, Norton MI, Van Horn JD, Wegner DM, Grafton ST, Macrae CN. 2007. Wandering minds: the default network and stimulus-independent thought. Science 315:393-395.

Medvedev ON, Siegert RJ, Feng XJ, Billington DR, Jang JY, Krägeloh CU. 2016. Measuring trait mindfulness: how to improve the precision of the Mindful Attention Awareness Scale using a Rasch Model. Mindfulness 7:384-395.

Menon V. 2015. Brain Mapping: An Encyclopedic Reference (Vol. 2). Waltham, MA: Academic Press.

Raichle ME. 2015. The brain's default mode network. Annu Rev Neurosci 38:433-447.

Raichle ME, MacLeod AM, Snyder AZ, Powers WJ, Gusnard DA, Shulman GL. 2001. A default mode of brain function. Proc Natl Acad Sci 98:676-682.

Rosazza C, Minati L. 2011. Resting-state brain networks: literature review and clinical applications. Neurol Sci 32:773-785.

Seeley WW, Menon V, Schatzberg AF, Keller J, Glover GH, Kenna H, Greicius MD. 2007. Dissociable intrinsic connec- tivity networks for salience processing and executive control. J Neurosci 27:2349-2356.

Shaurya Prakash R, De Leon AA, Klatt M, Malarkey W, Patterson B. 2013. Mindfulness disposition and default-mode network connectivity in older adults. Soc Cogn Affect Neurosci 8: 112-117.

Shin HS, Black DS, Shonkoff ET, Riggs NR, Pentz MA. 2016. Associations among dispositional mindfulness, self-compassion, and executive function proficiency in early adolescents. Mindfulness (N Y) 7:1377-1384.

Shirer WR, Ryali S, Rykhlevskaia E, Menon V, Greicius MD. 2012. Decoding subject-driven cognitive states with wholebrain connectivity patterns. Cereb Cortex 22:158-165.

Smallwood J, Schooler JW. 2006. The restless mind. Psychol Bull 132:946-958.

Smallwood J, Schooler JW. 2015. The science of mind wandering: empirically navigating the stream of consciousness. Annu Rev Psychol 66:487-518.

Sridharan D, Levitin DJ, Menon V. 2008. A critical role for the right fronto-insular cortex in switching between centralexecutive and default-mode networks. Proc Natl Acad Sci U S A 105:12569-12574.

Tanay G, Bernstein A. 2013. State Mindfulness Scale (SMS): development and initial validation. Psychol Assess 25: 1286-1299.

Tang YY, Lu Q, Fan M, Yang Y, Posner MI. 2012. Mechanisms of white matter changes induced by meditation. Proc Natl Acad Sci U S A 109:10570-10574.

Tang YY, Ma Y, Fan Y, Feng H, Wang J, Feng S, et al. 2009. Central and autonomic nervous system interaction is altered by shortterm meditation. Proc Natl Acad Sci U S A 106:8865-8870.

Tang YY, Ma Y, Wang J, Fan Y, Feng S, Lu Q, et al. 2007. Short-term meditation training improves attention and selfregulation. Proc Natl Acad Sci U S A 104:17152-17156.

Thera N. 2014. The Heart of Buddhist Meditation: The Buddha's Way of Mindfulness. San Francisco, California: Weiser Books.

Van Dam NT, Earleywine M, Borders A. 2010. Measuring mindfulness? An Item Response Theory analysis of the Mindful Attention Awareness Scale. Pers Individ Differ 49: 805-810.

Veen Vv, Carter CS. 2006. Conflict and cognitive control in the brain. Curr Dir Psychol Sci 15:237-240.

Vogt BA, Laureys S. 2005. Posterior cingulate, precuneal and retrosplenial cortices: cytology and components of the neural network correlates of consciousness. Prog Brain Res 150: 205-217.

Wang X, Xu M, Song Y, Li X, Zhen Z, Yang Z, Liu J. 2014. The network property of the thalamus in the default mode network is correlated with trait mindfulness. Neuroscience 278:291-301.

Zeidan F, Emerson NM, Farris SR, Ray JN, Jung Y, McHaffie JG, Coghill RC. 2015. Mindfulness meditation-based pain relief employs different neural mechanisms than placebo and sham mindfulness meditation-induced analgesia. J Neurosci 35:15307-15325.

\section{Address correspondence to: Jennifer Kornelsen Department of Radiology University of Manitoba GA216-820 Sherbrook Street Winnipeg, MB R3T 2N2 Canada}

E-mail: jennifer.kornelsen@umanitoba.ca 